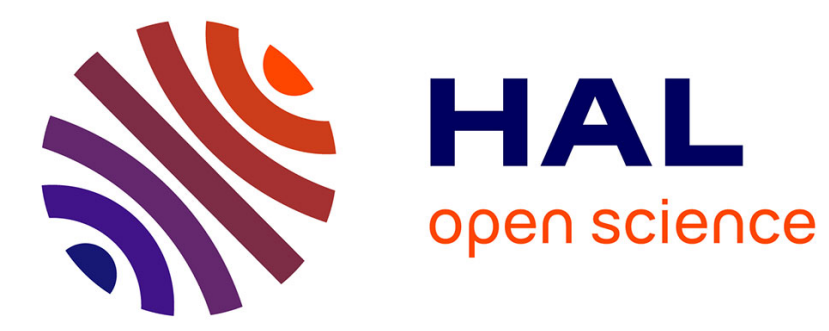

\title{
Epidemiology, outcome and types of invasive group A streptococcal infections in Finland
}

T. Siljander, O. Lyytikäinen, S. Vähäkuopus, M. Snellman, J. Jalava, J. Vuopio

\section{- To cite this version:}

T. Siljander, O. Lyytikäinen, S. Vähäkuopus, M. Snellman, J. Jalava, et al.. Epidemiology, outcome and types of invasive group A streptococcal infections in Finland. European Journal of Clinical Microbiology and Infectious Diseases, 2010, 29 (10), pp.1229-1235. 10.1007/s10096-010-0989-9 . hal00601637

\section{HAL Id: hal-00601637 \\ https://hal.science/hal-00601637}

Submitted on 20 Jun 2011

HAL is a multi-disciplinary open access archive for the deposit and dissemination of scientific research documents, whether they are published or not. The documents may come from teaching and research institutions in France or abroad, or from public or private research centers.
L'archive ouverte pluridisciplinaire HAL, est destinée au dépôt et à la diffusion de documents scientifiques de niveau recherche, publiés ou non, émanant des établissements d'enseignement et de recherche français ou étrangers, des laboratoires publics ou privés. 
Diseases

Editorial Manager(tm) for European Journal of Clinical Microbiology \& Infectious Manuscript Draft

Manuscript Number: EJCMID-D-10-00104R1

Title: Epidemiology, outcome and emm types of invasive group A streptococcal infections in Finland Article Type: Article

Keywords: group A streptococcus; Streptococcus pyogenes; epidemiology; emm typing; outcome; bacteraemia

Corresponding Author: Dr. Tuula Siljander, Ph.D.

Corresponding Author's Institution: National Institute for Health and Welfare

First Author: Tuula Siljander, Ph.D.

Order of Authors: Tuula Siljander, Ph.D.; Outi Lyytikäinen, M.D., Ph.D.; Susanna Vähäkuopus, M.Sc.; Marja Snellman, M.Sc.; Jari Jalava, Ph.D.; Jaana Vuopio, M.D., Ph.D.

Abstract: Purpose. In 2006, Finnish nationwide surveillance showed an increase of invasive group A streptococcal (iGAS) disease and clinicians were alarmed by severe disease manifestations, prompting investigation of recent trends and outcome for iGAS.

Methods. A case of iGAS was defined as Streptococcus pyogenes isolated from blood or cerebrospinal fluid. Cases during 1998-2007 and isolates during 2004-2007 were included. Case-patients' 7-day outcome was available for 2004-2007. Isolates were emm-typed.

Results. 1318 cases of iGAS were identified. The average annual incidence was 2.5/100,000 population. The rate was higher in males than females in persons aged 45-64 years, but lower in persons aged 2534 years. The annual incidence was highest in $2007(3.9 / 100,000)$. Occasional peaks occurred during midwinter and midsummer. The most common emm types were $28(21 \%), 1(16 \%), 84(10 \%), 75(7 \%)$ and 89 (6\%). During 2004-2007, emm1 replaced emm28 as the most predominant type. The overall case fatality was $8 \%$. Cases with emm 1 were associated with high case fatality $(14 \% \mathrm{vs} .8 \%$ in other types; $\mathrm{P}<0.02)$; that of emm28 infections was $2 \%(\mathrm{P}<0.01)$.

Conclusions. Changes in emm-type prevalence influenced incidence and case fatality. Differences in age- and sex-specific incidence and seasonal patterns suggest variation in predisposing factors and underlying conditions.

Response to Reviewers: European Journal of Clinical Microbiology \& Infectious Diseases

Response to reviewer comments

Manuscript No. EJCMID-D-10-00104: "Epidemiology, outcome and emm types of invasive group A streptococcal infections in Finland".

Reviewers' comments:

Reviewer \#1: The manuscript titled "Epidemiology, outcome and emm types of invasive group A streptococcal infections in Finland", by Siljander and colleagues, is a nicely performed analysis and 
description of surveillance for invasive GAS disease (iGAS) in Finland from 1998-2007. Their study and conclusion, that the changes in emm prevalence influenced iGAS incidence and case fatality ratios, add important information to the understanding o the epidemiology of iGAS disease. The analysis is appropriate and writing is well-organized, methodical and straightforward. This reviewer recommends that this manuscript be accepted for publication following several changes.

Response: Thank you for very relevant and constructive criticism towards this manuscript. Please find below a point-by-point response to the specific comments.

Specific comments:

Background and Methods are nicely detailed and clear.

Results: \% collection of isolates in recent years is quite amazing. Recommend that authors include \# of cases and rate by year; simple table form would be easiest.

Response: We added a new table (Table 1) to present the rate and number of cases and isolates by year and renumbered other tables correspondingly as Table 2 and 3. In addition, we changed the line spacing of all tables to improve readability.

Tables: nice to see the rate ratios by sex (table 1).

Fig b: how does the seasonality of cases by sex look per year? A large outbreak could distort the findings as shown in Fig b, appearing to be a repeated seasonal difference between men and women that doesn't actually exist each year.

Response: It is true that the seasonal patterns vary by year. We have to remember that our observations are based on very small numbers (3-23 cases per month). During the study, we did not detect any major outbreaks (within time/region/emm type), except for a small increase in cases caused by type emm84 (Siljander et al. 2009. J Clin Microbiol 47:477-480).

Please see below Figure A showing seasonality by sex per year. We decided to exclude this figure from the manuscript as it seemed too busy to read.

Figure A:

The summer peak occurred for males during 6/10 study years but for females only during 2/10 study years. We decided to slightly reformulate our statements in the manuscript as follows:

Results (page 5, lines 105-107): "Variation in the seasonal activity of iGAS disease could be identified, with occasional peaks of cases occurring during the midwinter (for both sexes) and midsummer (with male cases dominating; Figure 1a and 1b)."

Discussion (page 8, lines 198-201): "Seasonal variation in iGAS disease was observed; in addition to a midwinter peak season, there were also occasional peaks during the midsummer. In the northern hemisphere, peaks of cases are generally observed during the winter and spring. There were also sexspecific differences in the peak seasons, with male cases dominating during the summer."

Discussion:

First paragraph is a bit weak; could be strengthened (this is not necessary but would make for a better read). 
Response: We moved the text containing the most interesting points and strengths of the study into this paragraph (page 6 lines 135-141): "This study presents a comprehensive description of the recent epidemiology and outcome of invasive group A streptococcal (Streptococcus pyogenes) disease with information on emm type prevalence in a well-defined population. The main strength of the study is the nationwide population-based surveillance and the good coverage of isolate collection. We describe how the incidence and case fatality of iGAS infections were influenced by changes in the emm type prevalence. Age- and sex-specific differences in the incidence, uncharacteristic seasonal patterns of infections, and aspects of vaccine coverage are also discussed."

Authors should briefly discuss/support (if true) consistency in surveillance methods over time. Could the increasing incidence be due to improved surveillance methods? For example, any changes in blood culture practices, any change in completeness of reporting by labs?

Response: It is true that changes in blood culture practises or in the reporting system may have had an effect on the results. The annual blood-culturing rate increased by one-third during 1995-2002 in Finland (Skogberg et al.2008. Epidemiol Infect 136:108-114). Skogberg et al. concluded that "the increase in bloodstream infection rates may have been due to more frequent blood culturing but was not associated with changes in the reporting system [from paper to electronic]or aetiology of blood stream infections".

We added the following sentences in the discussion (page 9, lines 205-207): "There are some limitations to this study. Firstly, some of the increase in the incidence may be due to more frequent blood culturing activity [ref. Skogberg et al. 2008. Epidemiol Infect 136:108-114]. It is also possible but less likely that the incidence would have been influenced by changes in the reporting system, which was established in 1995."

We also detected regional differences in the blood-culturing rates. This was acknowledged in the discussion (page 9, lines 210-211): "Thirdly, differences in blood culture sampling practices between countries may have an effect on the observed incidence [ref. Skogberg et al. 2008. Epidemiol Infect 136:108-114]."

In addition, minor modifications were made in the paragraph regarding the limitations of this study to improve the discussion (page 9, lines 205-218).

Good to see comparisons to other European countries since this country is rather small and possibly not representative of a larger geographic area.

Why are there two seasonal peaks? Any theories?

Response: Without supporting evidence and data, we could not discuss our theories of the dual peak seasonality in the manuscript. The timing of the winter peak was somewhat earlier but near the expected peak based on data by other countries, and as it occurred for both sexes, we did not specifically question it. However, the summer peak differed from expectations on seasonality of these infections.

Factors associated with the summer peak could be the summer vacation period of Finns with the increased outdoor activities and possible risk behaviour. Alcohol-related diseases have been identified among the most common risk factors for invasive pneumococcal disease (Klemets et al. 2008. BMC Infect Dis 8: 96.) Exposure of the skin to insect bites and minor trauma may lead to skin infections and eventually invasive infections, and the higher temperature and humidity may delay the healing of wounds. Males and females may have different predisposing factors and underlying conditions and behavioural patterns. Males may also have a higher threshold than females for seeking medical care in case of trauma. 
We concluded that presumably the predisposing factors and underlying conditions associated with these peak seasons were different. Without data on all these above mentioned factors, we could not draw further conclusions on the reasons for seasonality. The small study size may cause some of the variation in the seasonality, which was discussed in the manuscript (pages 8-9, lines 202-204): "However, the unusual pattern and irregularity of the seasonality observed in this study may partly arise from natural variation due to a reasonably small study size."

Although it has been shown that emm types 1 and 3 are associated with increased mortality than other serotypes after controlling for age and underlying diseases, the authors should include this in their discussion. Although this surveillance does not capture other medical conditions, the authors could also state (if true) that emm 1 CFR is still higher when adjusted for age (for elderly) to strengthen argument.

Response: We agree with the above. However, in addition to the patient age, the underlying conditions of a patient also have a role in the case fatality. Without the clinical data, and with this small a study size, we did not adjust the case fatality of emm 1 infections for age. Nevertheless, to discuss this in the manuscript, we added the following sentences (page 8, lines 194-197): "Having an infection by emm type 1 or 3 has been identified as an independent factor associated with death due to iGAS disease [ref. O'Loughlin et al. 2007 Clin Infect Dis 45:853-62]. Also in our study, the case fatality by emm1 was higher than average, however we did not adjust it for age."

Page 7, line 170: add \% of 26-valent coverage for US and Japan.

Response: We modified the sentence as follows (page 7, lines 171-173): "The emm types included in the putative 26-valent recombinant vaccine would have covered approximately half of the Finnish isolates in 2004-2007, a notably smaller proportion than has been estimated for the U.S.A. (79\%) and Japan (82\%)."

Any documented support for the idea of strain competition? Couldn't this just reflect changes in general community immunity?

Response: It is true that we do not have sufficient evidence for strain competition because the time of surveillance and strain typing is not long enough to allow for that. We agree that it may be more accurate to stick to the concept of population immunity, whereas strain competition may only be part of that concept. We altered the statement as follows (page 8, lines 177-178): "Such a pattern of alternating type prevalence may be a reflection of changes in the general immunity of the population."

It has been speculated that the fluctuation in the prevalence of types reflects the population's susceptibility but also the prevalence of circulating types (mostly associated with mild infections and carriage of which we lack data) in the community. Regarding the idea of strain competition, the changing prevalence of emm 1 and emm 28 in Finland is of interest. Please see Figure B below (unpublished data; the coverage of surveillance may differ during the time period). The alternating prevalence of these types might suggest a competitive situation between these two types.

Figure B.

Page 8: lines 188-189: where did this 4-fold increase in case fatality come from? These numbers, if true, really need to be presented in the results or tables. This reviewer cannot find the data in the paper and 
questions the accuracy. Did the CFR reach $32 \%$ in 2005? Please check and correct! Or, rewrite this section to be more clear.

Response: We agree that the data was presented only partly which may distract the reader. The fourfold increase in the case fatality is true, but the statement needs more clarity to avoid misunderstanding. We modified the sentences regarding the case fatality as follows:

Results (pages 5-6, lines 122-123): "The case fatality was the lowest in 2004 (3\%), increasing to its highest in 2005 (12\%), and then declined by 2006 (10\%) and 2007 (7\%)."

Discussion (page 8, lines 191-192): "A sudden four-fold increase in the case fatality occurred from 3\% in 2004 to $12 \%$ in $2005 . "$ 
Click here to download Electronic Supplementary Material: Siljander_cover_letter_rev1.doc 


\section{European Journal of Clinical Microbiology \& Infectious Diseases}

\section{Response to reviewer comments}

Manuscript No. EJCMID-D-10-00104: "Epidemiology, outcome and emm types of invasive group A streptococcal infections in Finland".

Reviewers' comments:

Reviewer \#1: The manuscript titled "Epidemiology, outcome and emm types of invasive group A streptococcal infections in Finland", by Siljander and colleagues, is a nicely performed analysis and description of surveillance for invasive GAS disease (iGAS) in Finland from 1998-2007. Their study and conclusion, that the changes in emm prevalence influenced iGAS incidence and case fatality ratios, add important information to the understanding o the epidemiology of iGAS disease. The analysis is appropriate and writing is well-organized, methodical and straightforward. This reviewer recommends that this manuscript be accepted for publication following several changes.

Response: Thank you for very relevant and constructive criticism towards this manuscript. Please find below a point-by-point response to the specific comments.

Specific comments:

Background and Methods are nicely detailed and clear.

Results: \% collection of isolates in recent years is quite amazing. Recommend that authors include \# of cases and rate by year; simple table form would be easiest.

Response: We added a new table (Table 1) to present the rate and number of cases and isolates by year and renumbered other tables correspondingly as Table 2 and 3 . In addition, we changed the line spacing of all tables to improve readability.

Tables: nice to see the rate ratios by sex (table 1).

Fig b: how does the seasonality of cases by sex look per year? A large outbreak could distort the findings as shown in Fig b, appearing to be a repeated seasonal difference between men and women that doesn't actually exist each year.

Response: It is true that the seasonal patterns vary by year. We have to remember that our observations are based on very small numbers (3-23 cases per month). During the study, we did not detect any major outbreaks (within time/region/emm type), except for a small increase in cases caused by type emm84 (Siljander et al. 2009. J Clin Microbiol 47:477-480).

Please see below Figure A showing seasonality by sex per year. We decided to exclude this figure from the manuscript as it seemed too busy to read. 
Figure A:

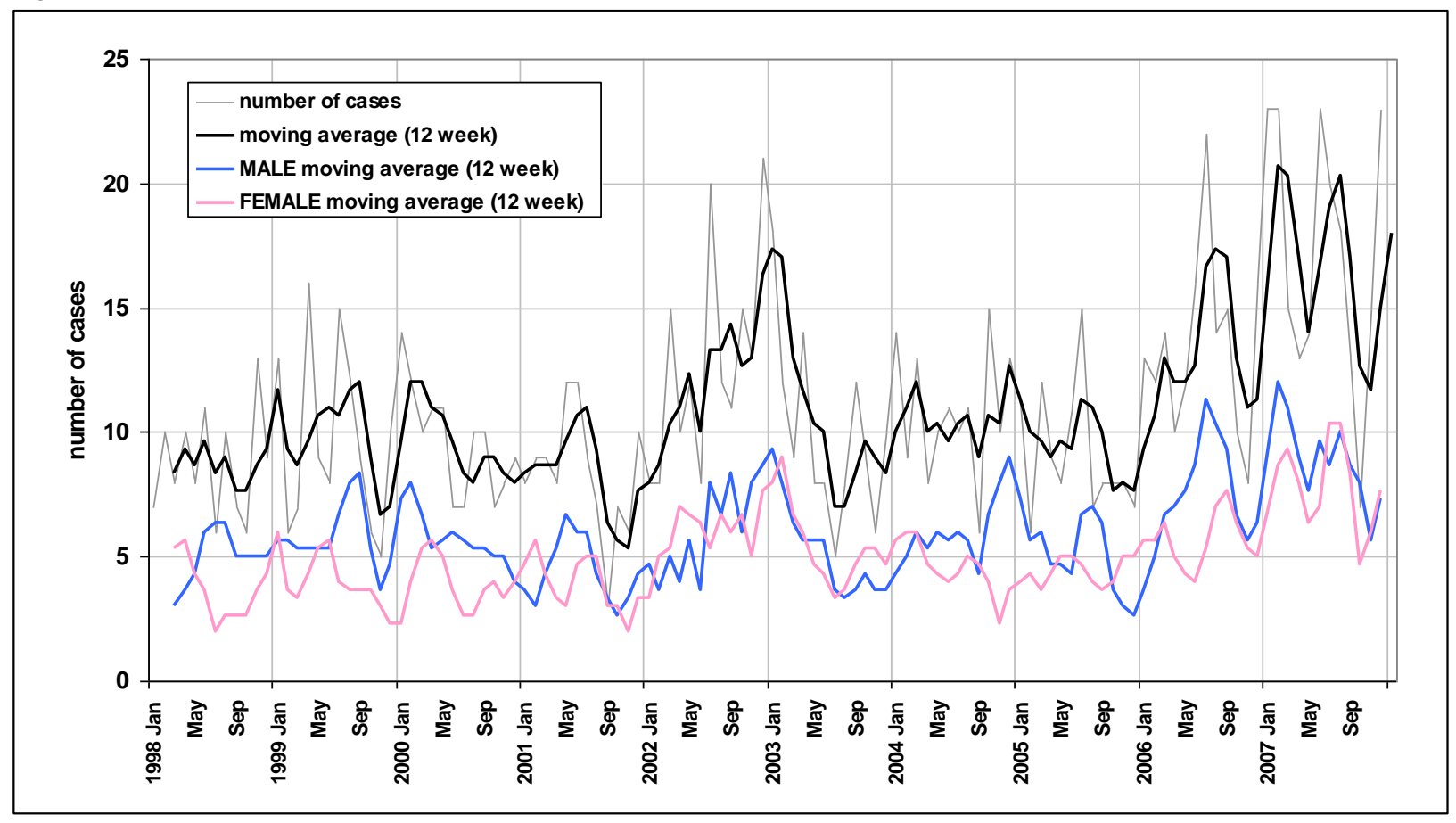

The summer peak occurred for males during 6/10 study years but for females only during 2/10 study years. We decided to slightly reformulate our statements in the manuscript as follows:

Results (page 5, lines 105-107): "Variation in the seasonal activity of iGAS disease could be identified, with occasional peaks of cases occurring during the midwinter (for both sexes) and midsummer (with male cases dominating; Figure la and $1 b$ )."

Discussion (page 8, lines 198-201): "Seasonal variation in iGAS disease was observed; in addition to a midwinter peak season, there were also occasional peaks during the midsummer. In the northern hemisphere, peaks of cases are generally observed during the winter and spring. There were also sex-specific differences in the peak seasons, with male cases dominating during the summer."

Discussion:

First paragraph is a bit weak; could be strengthened (this is not necessary but would make for a better read).

Response: We moved the text containing the most interesting points and strengths of the study into this paragraph (page 6 lines 135-141): "This study presents a comprehensive description of the recent epidemiology and outcome of invasive group A streptococcal (Streptococcus pyogenes) disease with information on emm type prevalence in a well-defined population. The main strength of the study is the nationwide population-based surveillance and the good coverage of isolate collection. We describe how the incidence and case fatality of iGAS infections were influenced by changes in the emm type prevalence. Age-and sex-specific differences in the incidence, uncharacteristic seasonal patterns of infections, and aspects of vaccine coverage are also discussed."

Authors should briefly discuss/support (if true) consistency in surveillance methods over time. Could the increasing incidence be due to improved surveillance methods? For example, any changes in blood culture practices, any change in completeness of reporting by labs? 
Response: It is true that changes in blood culture practises or in the reporting system may have had an effect on the results. The annual blood-culturing rate increased by one-third during 1995-2002 in Finland (Skogberg et al.2008. Epidemiol Infect 136:108-114). Skogberg et al. concluded that "the increase in bloodstream infection rates may have been due to more frequent blood culturing but was not associated with changes in the reporting system [from paper to electronic]or aetiology of blood stream infections".

We added the following sentences in the discussion (page 9, lines 205-207): "There are some limitations to this study. Firstly, some of the increase in the incidence may be due to more frequent blood culturing activity [ref. Skogberg et al. 2008. Epidemiol Infect 136:108-114]. It is also possible but less likely that the incidence would have been influenced by changes in the reporting system, which was established in 1995."

We also detected regional differences in the blood-culturing rates. This was acknowledged in the discussion (page 9, lines 210-211): "Thirdly, differences in blood culture sampling practices between countries may have an effect on the observed incidence [ref. Skogberg et al. 2008. Epidemiol Infect 136:108-114]."

In addition, minor modifications were made in the paragraph regarding the limitations of this study to improve the discussion (page 9, lines 205-218).

Good to see comparisons to other European countries since this country is rather small and possibly not representative of a larger geographic area.

Why are there two seasonal peaks? Any theories?

Response: Without supporting evidence and data, we could not discuss our theories of the dual peak seasonality in the manuscript. The timing of the winter peak was somewhat earlier but near the expected peak based on data by other countries, and as it occurred for both sexes, we did not specifically question it. However, the summer peak differed from expectations on seasonality of these infections.

Factors associated with the summer peak could be the summer vacation period of Finns with the increased outdoor activities and possible risk behaviour. Alcohol-related diseases have been identified among the most common risk factors for invasive pneumococcal disease (Klemets et al. 2008. BMC Infect Dis 8: 96.) Exposure of the skin to insect bites and minor trauma may lead to skin infections and eventually invasive infections, and the higher temperature and humidity may delay the healing of wounds. Males and females may have different predisposing factors and underlying conditions and behavioural patterns. Males may also have a higher threshold than females for seeking medical care in case of trauma.

We concluded that presumably the predisposing factors and underlying conditions associated with these peak seasons were different. Without data on all these above mentioned factors, we could not draw further conclusions on the reasons for seasonality. The small study size may cause some of the variation in the seasonality, which was discussed in the manuscript (pages 8-9, lines 202-204):

"However, the unusual pattern and irregularity of the seasonality observed in this study may partly arise from natural variation due to a reasonably small study size."

Although it has been shown that emm types 1 and 3 are associated with increased mortality than other serotypes after controlling for age and underlying diseases, the authors should include this in their discussion. Although this surveillance does not capture other medical conditions, the authors could also state (if true) that emm 1 CFR is still higher when adjusted for age (for elderly) to strengthen argument.

Response: We agree with the above. However, in addition to the patient age, the underlying conditions of a patient also have a role in the case fatality. Without the clinical data, and with this 
small a study size, we did not adjust the case fatality of $\mathrm{emml}$ infections for age. Nevertheless, to discuss this in the manuscript, we added the following sentences (page 8, lines 194-197): "Having an infection by emm type 1 or 3 has been identified as an independent factor associated with death due to iGAS disease [ref. O'Loughlin et al. 2007 Clin Infect Dis 45:853-62]. Also in our study, the case fatality by emml was higher than average, however we did not adjust it for age."

Page 7, line 170: add \% of 26-valent coverage for US and Japan.

Response: We modified the sentence as follows (page 7, lines 171-173): "The emm types included in the putative 26-valent recombinant vaccine would have covered approximately half of the Finnish isolates in 2004-2007, a notably smaller proportion than has been estimated for the U.S.A. (79\%) and Japan (82\%).”

Any documented support for the idea of strain competition? Couldn't this just reflect changes in general community immunity?

Response: It is true that we do not have sufficient evidence for strain competition because the time of surveillance and strain typing is not long enough to allow for that. We agree that it may be more accurate to stick to the concept of population immunity, whereas strain competition may only be part of that concept. We altered the statement as follows (page 8, lines 177-178): "Such a pattern of alternating type prevalence may be a reflection of changes in the general immunity of the population."

It has been speculated that the fluctuation in the prevalence of types reflects the population's susceptibility but also the prevalence of circulating types (mostly associated with mild infections and carriage of which we lack data) in the community. Regarding the idea of strain competition, the changing prevalence of emml and emm 28 in Finland is of interest. Please see Figure B below (unpublished data; the coverage of surveillance may differ during the time period). The alternating prevalence of these types might suggest a competitive situation between these two types.

Figure B.

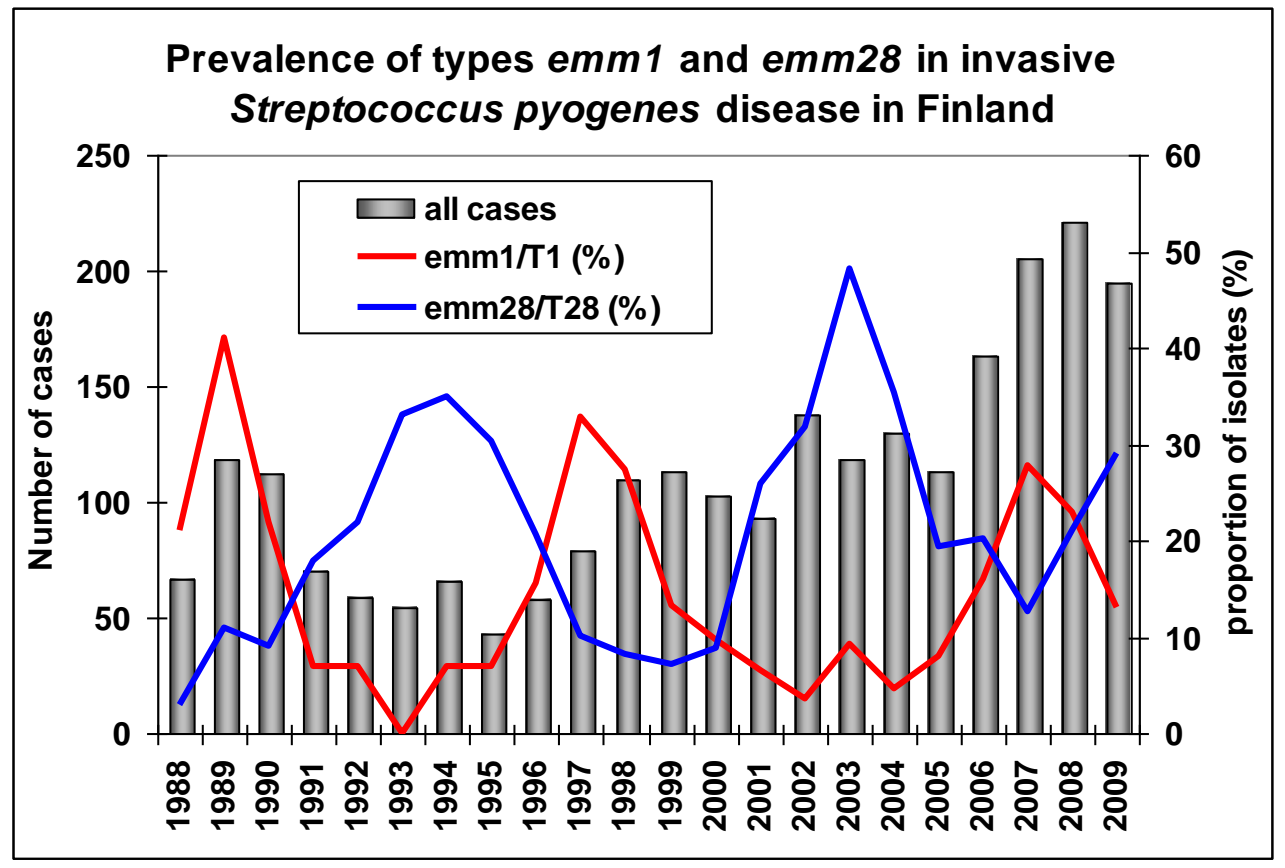

Page 8: lines 188-189: where did this 4-fold increase in case fatality come from? These numbers, if true, really need to be presented in the results or tables. This reviewer cannot find the data in the paper and 
questions the accuracy. Did the CFR reach 32\% in 2005? Please check and correct! Or, rewrite this section to be more clear.

Response: We agree that the data was presented only partly which may distract the reader. The four-fold increase in the case fatality is true, but the statement needs more clarity to avoid misunderstanding. We modified the sentences regarding the case fatality as follows:

Results (pages 5-6, lines 122-123): "The case fatality was the lowest in 2004 (3\%), increasing to its highest in 2005 (12\%), and then declined by 2006 (10\%) and 2007 (7\%)."

Discussion (page 8, lines 191-192): "A sudden four-fold increase in the case fatality occurred from $3 \%$ in 2004 to $12 \%$ in 2005." 
Epidemiology, outcome and emm types of invasive group A streptococcal infections in Finland

2 Tuula Siljander ${ }^{1}$, Outi Lyytikäinen ${ }^{2}$, Susanna Vähäkuopus ${ }^{1}$, Marja Snellman ${ }^{2}$, Jari Jalava ${ }^{3}$, Jaana

3 Vuopio $^{1}$

4

$5 \quad{ }^{1}$ Bacteriology Unit, Department of Infectious Disease Surveillance and Control, National Institute for

6 Health and Welfare, P.O. Box 30, FI-00271, Helsinki, Finland

$7 \quad{ }^{2}$ Epidemiologic Surveillance and Response Unit, Department of Infectious Disease Surveillance and

8 Control, National Institute for Health and Welfare, P.O. Box 30, FI-00271, Helsinki, Finland

$9{ }^{3}$ Antimicrobial Resistance Unit, Department of Infectious Disease Surveillance and Control, National

10 Institute for Health and Welfare, Kiinamyllynkatu 13, FIN-20521, Turku, Finland

11

12 Running title: Invasive group A streptococcus, Finland

13

14 Corresponding author: Tuula Siljander, Department of Infectious Disease Surveillance and Control,

15 National Institute for Health and Welfare, P.O. Box 30, FI-00271, Helsinki, Finland. E-mail:

16 tuula.siljander@thl.fi, phone: +358 20610 8558, fax: +358 206108238 ,

19 Category:

20 Word count for abstract: 200

21 Word count for text: 2347

22 Number of figures: 1

23 Number of tables: 3 
ABSTRACT

26 Purpose. In 2006, Finnish nationwide surveillance showed an increase of invasive group A

27 streptococcal (iGAS) disease and clinicians were alarmed by severe disease manifestations, prompting 28 investigation of recent trends and outcome for iGAS.

29 Methods. A case of iGAS was defined as Streptococcus pyogenes isolated from blood or 30 cerebrospinal fluid. Cases during 1998-2007 and isolates during 2004-2007 were included. Case31 patients' 7-day outcome was available for 2004-2007. Isolates were emm-typed.

32 Results. 1318 cases of iGAS were identified. The average annual incidence was 2.5/100,000 33 population. The rate was higher in males than females in persons aged $45-64$ years, but lower in 34 persons aged 25-34 years. The annual incidence was highest in 2007 (3.9/100,000). Occasional peaks 35 occurred during midwinter and midsummer. The most common emm types were $28(21 \%), 1(16 \%)$, $3684(10 \%), 75(7 \%)$ and $89(6 \%)$. During 2004-2007, emm 1 replaced emm 28 as the most predominant 37 type. The overall case fatality was $8 \%$. Cases with emm 1 were associated with high case fatality (14\% 38 vs. $8 \%$ in other types; $\mathrm{P}<0.02)$; that of $e m m 28$ infections was $2 \%(\mathrm{P}<0.01)$.

39 Conclusions. Changes in emm-type prevalence influenced incidence and case fatality. Differences in 40 age- and sex-specific incidence and seasonal patterns suggest variation in predisposing factors and 41 underlying conditions.

43 Key words: group A streptococcus; Streptococcus pyogenes; epidemiology; emm typing; outcome;

44 bacteraemia 
Streptococcus pyogenes (group A streptococcus, GAS) causes a wide array of infections ranging in severity from mild pharyngitis and skin/soft tissue infections to severe invasive infections. In the late 1980s, a change in the epidemiology of severe GAS infections, with an increase in the incidence and severity of disease was documented [20]. The role and increased pathogenic potential of type M1 in epidemics were of specific interest $[13,25]$. The incidence of severe GAS disease shows variations over time and geographic region, possibly reflecting the population's susceptibility to particular strains with diverse virulence properties $[9,17,26]$. Dynamic changes in the type distribution of GAS may also influence the case fatality of these infections.

In Finland, the incidence rate of invasive group A streptococcal (iGAS) disease has also shown fluctuations during this and the previous decade, and an increasing trend has been noticed [21]. In 2006, clinicians contacted the National Institute for Health and Welfare and described suddenly encountering more severe disease manifestations with a poor outcome. These events prompted us to investigate the recent trend and outcome of iGAS infections and emm type distribution in Finland in more detail.

\section{METHODS}

Surveillance. The national healthcare system of Finland (population 5.3 million) is organised into 20 healthcare districts (with catchment populations ranging from 58,000 to 1.5 million), forming five tertiary care districts. Since 1995, all clinical microbiology laboratories have mandatorily notified all isolations of S. pyogenes from blood or cerebrospinal fluid (CSF) to the National Infectious Disease Register (NIDR). With each notification, the following information is transmitted (generally electronically) to the NIDR: date and type of specimen, unique national identity code of the patient (since 2004), date of birth, sex, and place of treatment. Within an interval of three months, notifications concerning the same patient are merged into a single case. The corresponding $S$. pyogenes isolates are submitted to the national reference laboratory. 
71 Case definition and outcome. A case of iGAS was defined as S. pyogenes isolated from blood or

72 CSF. In this study, iGAS cases from January 1998 to December 2007 and isolates from January 2004

73 to December 2007 were included. Case-patients' vital status at 7 days of positive blood or CSF culture 74 was obtained for 2004-2007 from the Population Information System through the use of the national 75 identity codes.

76 Identification and characterisation of isolates. Isolates were tested for sensitivity to bacitracin, and 77 the Lancefield group A antigen was identified using Streptex latex agglutination (Remel Europe Ltd, 78 U.K.). emm typing was performed for all isolates according to guidelines by the Centers of Disease 79 Control and Prevention (http://www.cdc.gov/ncidod/biotech/strep/protocol_emm-type.htm) as 80 previously described [22]. Susceptibility to erythromycin, clindamycin, and tetracycline was 81 determined with agar dilution method for all isolates. Susceptibility was assigned according to 82 interpretative criteria for MIC as recommended by the Clinical and Laboratory Standards Institute [2].

83 Data analysis and statistics. Data on the cases of iGAS, their outcome, and emm typing and 84 antimicrobial susceptibility data (where available) were linked using the national identity code of the 85 case-patient and the date of specimen. Where the national identity code was unavailable, other 86 available information was used for linkage. Average annual incidence rates were calculated for 1998872007 using the population census at the end of the previous year, as obtained from Statistics Finland. 88 Age- and sex-specific incidence rates and male-to-female ratios with 95\% confidence intervals (CI) 89 according to Poisson distribution were calculated. 7-day case fatality was calculated for 2004-2007 90 and for each emm type separately. $\chi^{2}$ and Kruskal-Wallis tests were applied where appropriate to test 91 for statistical significance between subgroups and distributions. Differences were considered 92 significant when $\mathrm{P}<0.05$. Data were analyzed with Intercooled Stata ${ }^{\mathrm{TM}} 9.1$ software for Windows 93 (StataCorp, U.S.) and R statistical software version 2.9.0 (R Development Core Team, Austria).

\section{RESULTS}

95 Incidence rates. From January 1998 to December 2007, 1318 cases of iGAS (range by year, 100-207) 96 were identified (Table 1). The median age of case-patients was 52 years (range, 0-95 years); 55\% were 97 males. The average annual incidence rate of iGAS was 2.5 cases per 100,000 population. The rate was 
98

higher in males than in females and in males of adult age groups it increased with age (Table 2). The highest rates were observed among the elderly patients. Males had a significantly higher rate of infection than females especially among persons aged 45-64 years, whereas females had a higher rate than males among persons aged 25-34 years.

The annual incidence rate fluctuated (range by year, 1.9-3.9 cases per 100,000 population) but showed an increasing trend during the study period. Peaks occurred in 2002 (2.9 cases per 100,000) and in 2006-2007 (3.1-3.9). The average annual incidence rate in the five tertiary care districts varied between 1.8-3.1 cases per 100,000. Of all cases, $41 \%$ were localized in the tertiary care district around Helsinki, where the annual incidence rate varied within the range of 2.2-4.4 cases per 100,000. Variation in the seasonal activity of iGAS disease could be identified, with occasional peaks of cases occurring during the midwinter (for both sexes) and midsummer (with male cases dominating; Figure $1 \mathrm{a}$ and $1 \mathrm{~b})$.

emm sequence types. For 602 out of 609 cases during 2004-2007, a corresponding isolate was received. A total of $46 \mathrm{emm}$ types were encountered (range by year, 23-28 types). The annual 112 proportion of non-typable isolates varied within $1-5 \%$. The five most common emm types were 28,1 , 11384,75 and 89, together accounting for $60 \%$ of isolates (Table 3). Type emm 28 was the most 114 predominant type in 2004 , but its proportion declined each year while that of $e m m 1$ increased, and by 1152007 , emm 1 became the most common type. Type emm 1 isolates were mainly of subtypes 1.0 and 1161.10 , but sporadic cases of other subtypes were also encountered. A new and uncommon type, emm84, 117 emerged in 2005 and became the second most common type by 2007.

118 Type emm28 infections were over-represented in females compared to males ( $55 \%$ vs. $45 \%, \mathrm{P}<0.01$ ), 119 and the same was true for emm 4 infections ( $73 \%$ vs. $27 \%$, respectively, $\mathrm{P}<0.05$ ). Specifically, females 120 of child-bearing age (15-44 years) had a significantly larger proportion of infections by emm 28 than 121 males of the same age ( $48 \%$ vs. $21 \%$ of emm 28 infections, respectively; $\mathrm{P}<0.01$ ).

122 Outcome. During 2004-2007, 48/609 case-patients died within 7 days of the positive blood or CSF 123 culture (overall case fatality, $8 \%$ ). The case fatality did not significantly differ between males and 124 females (9\% vs. 6\%, P=0.172). The case fatality was the lowest in 2004 (3\%), increasing to its highest 
in 2005 (12\%), and then declined by 2006 (10\%) and 2007 (7\%). The case-patients with fatal outcome

1126

2

were older than those who survived (median age, 60 vs. 54 years; $\mathrm{P}<0.01$ ). No fatalities occurred in patients aged $<1$ and 15-24 years.

Overall, infections by emm 1 were significantly associated with higher case fatality (14\% vs. $8 \%$ with all other types; $\mathrm{P}<0.02$, Table 3 ). Cases by emm 75 and 79 were also associated with higher than average case fatality (14\% for both). In contrast, emm 28 was associated with lower case fatality ( $2 \%$ vs. all other types, $\mathrm{P}<0.01)$ along with type $81(0 \%$, respectively).

Antimicrobial susceptibility. Of the iGAS isolates tested, $1.5 \%(9 / 602)$ were resistant to erythromycin, $0.5 \%$ (3/602) to clindamycin and $16 \%(97 / 602)$ to tetracycline. The tetracycline resistant strains were mainly of emm types 81 (20 resistant isolates in total; $87 \%$ of this type resistant to tetracycline), $27 \mathrm{G}(19 ; 100 \%), 77(12 ; 100 \%)$ and $85(9 ; 100 \%)$.

\section{DISCUSSION}

137 This study presents a comprehensive description of the recent epidemiology and outcome of invasive group A streptococcal (Streptococcus pyogenes) disease with information on emm type prevalence in a well-defined population. The main strength of the study is the nationwide population-based surveillance and the good coverage of isolate collection. We describe how the incidence and case fatality of iGAS infections were influenced by changes in the emm type prevalence. Age- and sexspecific differences in the incidence, uncharacteristic seasonal patterns of infections, and aspects of vaccine coverage are also discussed.

The observed incidence rate of 3.9 cases per 100,000 population in 2007 is the highest in Finland since 1995. This study together with our earlier research shows that the incidence of iGAS disease has fluctuated since the 1990s but continued its increasing trend [21, 23]. During 2003-2004, the U.K., Sweden, Denmark and Finland had the highest rates of infection (range 2.5-3.3 per 100,000) in a European study [9]. Since then, indications of an increase in the incidence are seen in Sweden to 4.5 cases per 100,000 by 2007 (http://www.smittskyddsinstitutet.se/in-english/statistics/beta-hemolyticgroup-a-streptococci-gas-invasiv-infection/) and very recently in the U.K. [11]. In contrast, the incidence of iGAS disease in the U.S.A. has remained relatively stable, at an average of 3.5 cases per 
1153 (http://www.cdc.gov/abcs/survreports.htm) [17].

The incidence of iGAS disease in Finland generally increased with age as expected, but the rate in the elderly ( $\geq 65$ years) still remained at a lower level than has been reported in Sweden, Denmark, the U.K. or the U.S.A. $[4,10,12,17]$. Males had a higher incidence of iGAS disease compared to females, age- and sex-specific rates, with males having significantly more infections than females in the age of 45-64 years. In contrast, females had more infections in the age of 25-34 years than males. A similar clear peak in the incidence of females around the same age has been noticed in earlier studies from Denmark and the U.K. [10, 12].

The characteristic fluctuation in the incidence of iGAS disease is presumably a reflection of changes in the emm type distribution and the population's susceptibility towards particular strains, especially of emerging types $[4,16,17]$. The emm type distribution in Finland underwent major changes during 2004-2007 when emm1 replaced emm28 as the most predominant type. The incidence of iGAS disease increased simultaneously with the increasing prevalence of type emm1, as has also been observed by others [25]. However, changes in the prevalence of other types, such as the sudden emergence of emm84, also contributed to the increase [23].

The type distribution of iGAS isolates in Finland has common features with that of other countries but also some unique characteristics. emm types 1,28 and 89 are common globally but 75 and 84 are rarer and have not featured among the five most common emm types elsewhere $[4,5,7,12,17,26]$. The low prevalence of cases by emm 3 is of interest, as it is a common type in many countries, and often associated with high case fatality $[7,12,17,26]$. The emm types included in the putative 26 -valent recombinant vaccine would have covered approximately half of the Finnish isolates in 2004-2007, a notably smaller proportion than has been estimated for the U.S.A. (79\%) and Japan (82\%) [7, 14, 17].

Within the last 20 years, a periodic fluctuation of peaks and troughs of the two most common genotypes emm 1 and emm 28 has occurred in Finland. Peaks in numbers of M/emm 1 have been observed earlier in 1988-1990 and 1997-1998, and in this study in 2006-2007 [6, 15, 21]. Similarly, 
the prevalence of type emm28 has peaked in 1993-1995 and 2002-2004 [15, 21]. Such a pattern of

alternating type prevalence may be a reflection of changes in the general immunity of the population.

Types emm 28 and more rarely also emm 4 have been known to associate with postpartum infections and puerperal sepsis $[1,3,26]$. Also in this study, emm28 infections were found to be specifically concentrated in females of child-bearing age. However, estimations of how many of these cases were pregnancy-related infections are beyond the scope of this study.

185 The overall erythromycin resistance during 2004-2007 was expectedly low, as the rate has declined 186 during this decade in Finland

187 (http://www.ktl.fi/portal/english/projects/fire/finres/finres_antimicrobial_resistance_statistics_/strept 188 ococcus_pyogenes/). The Finnish iGAS strains remain susceptible to clindamycin. Similar low 189 resistance rates have been reported for example in Denmark and the U.S.A. [12, 19]. Tetracycline 190 resistance was associated with certain clones, as has also been found previously, and the resistant 191 strains were mainly of uncommon emm types [8].

192 The overall case fatality of iGAS disease in Finland was relatively low at $8 \%$, compared to those 193 reported by some other European countries and the U.S.A. [4, 5, 10, 17]. A sudden four-fold increase 194 in the case fatality occurred from $3 \%$ in 2004 to $12 \%$ in 2005 . During the study, the proportion of 195 infections by types $\mathrm{emm} 1$ and $\mathrm{emm} 75$ increased, these types being associated with a high case fatality. 196 In parallel, the proportion of emm28, associated with a low case fatality, decreased. Having an 197 infection by emm type 1 or 3 has been identified as an independent factor associated with death due to 198 iGAS disease [17]. Also in our study, the case fatality by emm 1 was higher than average, however we 199 did not adjust it for age.

200 Seasonal variation in iGAS disease was observed; in addition to a midwinter peak season, there were 201 also occasional peaks during the midsummer. In the northern hemisphere, peaks of cases are generally 202 observed during the winter and spring $[4,9,17,26]$. There were also sex-specific differences in the peak seasons, with male cases dominating during the summer. These two peak seasons might be associated with different predisposing factors and underlying conditions. However, the unusual pattern 
and irregularity of the seasonality observed in this study may partly arise from natural variation due to

1206 a reasonably small study size.

2

3

There are some limitations to this study. Firstly, some of the increase in the incidence may be due to more frequent blood culturing activity [24]. It is also possible but less likely that the incidence would have been influenced by changes in the reporting system, which was established in 1995. Secondly, case definitions of iGAS disease differ between countries and complicate comparisons of incidence rates. Exclusion of non-bacteraemic cases with other sterile site isolations and/or toxic shock by the Finnish case definition may partly explain the observed lower incidence rate. Thirdly, differences in blood culture sampling practices between countries may have an effect on the observed incidence [24]. In addition, the observed differences in fatality may arise from the case definition used, the timing of death considered (7/30-day/in-hospital mortality), and the source of information (e.g. population registry vs. hospital records). In this study, the 7-day mortality information obtained from the Population Information System was used. Finally, the surveillance does not include collection of clinical data of disease manifestations, possible risk factors and underlying conditions of patients. These data would be needed to address reasons behind the observed differences in the age-and sexspecific incidence, and such a study has been undertaken within one healthcare district in Finland [18].

221 In conclusion, dynamic changes in the emm type prevalence influenced the incidence and case fatality 222 of iGAS disease in Finland. An unusual seasonal pattern with peaks in the midwinter and midsummer 223 was observed. Differences in age- and sex-specific incidence rates and seasonality suggest variation in predisposing factors and underlying conditions. 


\section{ACKNOWLEDGEMENTS}

1
Laboratory technicians Aila Soininen, Saija Perovuo, Suvi Kavenius, Tuula Randell and Minna

Lamppu are greatly acknowledged for excellent technical assistance. Data Manager Joonas Iivonen is acknowledged for technical expertise with retrieving and combining data from several database sources. Clinical microbiology laboratories are acknowledged for submitting notifications and isolates.

Previous presentations: These results have partly been presented at the European Scientific

Conference on Applied Infectious Disease Epidemiology (ESCAIDE), October 2007, Stockholm, 233 Sweden (poster number B.A.1.28), at the XVII Lancefield International Symposium on Streptococci 234 and Streptococcal Diseases (LISSSD), June 2008, Porto Heli, Greece (abstract number O 4.4), and at 235 the European Scientific Conference on Applied Infectious Disease Epidemiology (ESCAIDE), 236 October 2009, Stockholm, Sweden (abstract number 20090261).

238 Funding sources: Funding was received from The Ministry of Social Affairs and Health, the Paulo 239 Foundation, the Sigrid Jusélius Foundation, the University of Helsinki, the Finnish Cultural 240 Foundation and the Alfred Kordelin Foundation.

242 Conflict of interest: The authors declare that they have no conflict of interest. 


\section{REFERENCES}

1. Chuang I, Van Beneden C, Beall B, Schuchat A (2002) Population-based surveillance for postpartum invasive group a streptococcus infections, 1995-2000. Clin Infect Dis 35:665-670

2. CLSI (2007) Clinical and Laboratory Standards Institute (CLSI). Performance standard for antimicrobial susceptibility testing; seventeenth informational supplement M100-S17, vol 24 no 1

3. Colman G, Tanna A, Efstratiou A, Gaworzewska ET (1993) The serotypes of Streptococcus pyogenes present in Britain during 1980-1990 and their association with disease. J Med Microbiol 39:165-178

4. Darenberg J, Luca-Harari B, Jasir A, Sandgren A, Pettersson H, Schalen C, Norgren M, Romanus V, Norrby-Teglund A, Normark BH (2007) Molecular and clinical characteristics of invasive group A streptococcal infection in Sweden. Clin Infect Dis 45:450-458

5. Ekelund K, Darenberg J, Norrby-Teglund A, Hoffmann S, Bang D, Skinhoj P, Konradsen HB (2005) Variations in emm type among group A streptococcal isolates causing invasive or noninvasive infections in a nationwide study. J Clin Microbiol 43:3101-3109

6. Hoe NP, Nakashima K, Lukomski S, Grigsby D, Liu M, Kordari P, Dou SJ, Pan X, VuopioVarkila J, Salmenlinna S, McGeer A, Low DE, Schwartz B, Schuchat A, Naidich S, De Lorenzo D, Fu YX, Musser JM (1999) Rapid selection of complement-inhibiting protein variants in group A Streptococcus epidemic waves. Nat Med 5:924-929

7. Ikebe T, Hirasawa K, Suzuki R, Ohya H, Isobe J, Tanaka D, Katsukawa C, Kawahara R, Tomita M, Ogata K, Endoh M, Okuno R, Tada Y, Okabe N, Watanabe H (2007) Distribution of emm genotypes among group A streptococcus isolates from patients with severe invasive streptococcal infections in Japan, 2001-2005. Epidemiol Infect 135:1227-1229

8. Kataja J, Huovinen P, Muotiala A, Vuopio-Varkila J, Efstratiou A, Hallas G, Seppala H (1998) Clonal spread of group A streptococcus with the new type of erythromycin resistance. Finnish Study Group for Antimicrobial Resistance. J Infect Dis 177:786-789

9. Lamagni TL, Darenberg J, Luca-Harari B, Siljander T, Efstratiou A, Henriques-Normark B, Vuopio-Varkila J, Bouvet A, Creti R, Ekelund K, Koliou M, Reinert RR, Stathi A, Strakova L, Ungureanu V, Schalen C, Jasir A (2008) Epidemiology of severe Streptococcus pyogenes disease in Europe. J Clin Microbiol 46:2359-2367

10. Lamagni TL, Neal S, Keshishian C, Alhaddad N, George R, Duckworth G, Vuopio-Varkila J, Efstratiou A (2008) Severe Streptococcus pyogenes infections, United Kingdom, 2003-2004. Emerg Infect Dis 14:202-209 
11. Lamagni TL, Efstratiou A, Dennis J, Nair P, Kearney J, George R (2009) Increase in invasive group A streptococcal infections in England, Wales and Northern Ireland, 2008-9. Euro Surveill 14

12. Luca-Harari B, Ekelund K, van der Linden M, Staum-Kaltoft M, Hammerum AM, Jasir A (2008) Clinical and epidemiological aspects of invasive Streptococcus pyogenes infections in Denmark during 2003 and 2004. J Clin Microbiol 46:79-86

13. Martin DR, Single LA (1993) Molecular epidemiology of group A streptococcus M type 1 infections. J Infect Dis 167:1112-1117

14. McNeil SA, Halperin SA, Langley JM, Smith B, Warren A, Sharratt GP, Baxendale DM, Reddish MA, Hu MC, Stroop SD, Linden J, Fries LF, Vink PE, Dale JB (2005) Safety and immunogenicity of 26-valent group A Streptococcus vaccine in healthy adult volunteers. Clin Infect Dis 41:11141122

15. Muotiala A, Seppala H, Huovinen P, Vuopio-Varkila J (1997) Molecular comparison of group A streptococci of T1M1 serotype from invasive and noninvasive infections in Finland. J Infect Dis 175:392-399

16. O'Brien KL, Beall B, Barrett NL, Cieslak PR, Reingold A, Farley MM, Danila R, Zell ER, Facklam R, Schwartz B, Schuchat A (2002) Epidemiology of invasive group A streptococcus disease in the United States, 1995-1999. Clin Infect Dis 35:268-276

17. O'Loughlin RE, Roberson A, Cieslak PR, Lynfield R, Gershman K, Craig A, Albanese BA, Farley MM, Barrett NL, Spina NL, Beall B, Harrison LH, Reingold A, Van Beneden C (2007) The epidemiology of invasive group A streptococcal infection and potential vaccine implications: United States, 2000-2004. Clin Infect Dis 45:853-862

18. Rantala S, Vuopio-Varkila J, Vuento R, Huhtala H, Syrjanen J (2009) Clinical presentations and epidemiology of beta-haemolytic streptococcal bacteraemia: a population-based study. Clin Microbiol Infect 15:286-288

19. Richter SS, Heilmann KP, Beekmann SE, Miller NJ, Miller AL, Rice CL, Doern CD, Reid SD, Doern GV (2005) Macrolide-resistant Streptococcus pyogenes in the United States, 2002-2003. Clin Infect Dis 41:599-608

20. Schwartz B, Facklam RR, Breiman RF (1990) Changing epidemiology of group A streptococcal infection in the USA. Lancet 336:1167-1171

21. Siljander T, Toropainen M, Muotiala A, Hoe NP, Musser JM, Vuopio-Varkila J (2006) emm typing of invasive T28 group A streptococci, 1995-2004, Finland. J Med Microbiol 55:1701-1706 
9

22. Siljander T, Karppelin M, Vahakuopus S, Syrjanen J, Toropainen M, Kere J, Vuento R, Jussila T, Vuopio-Varkila J (2008) Acute bacterial, nonnecrotizing cellulitis in Finland: microbiological findings. Clin Infect Dis 46:855-861

23. Siljander T, Lyytikainen O, Vahakuopus S, Saila P, Jalava J, Vuopio-Varkila J (2009) Rapid emergence of emm84 among invasive Streptococcus pyogenes infections in Finland. J Clin Microbiol 47:477-480

24. Skogberg K, Lyytikainen O, Ruutu P, Ollgren J, Nuorti JP (2008) Increase in bloodstream infections in Finland, 1995-2002. Epidemiol Infect 136:108-114

25. Stromberg A, Romanus V, Burman LG (1991) Outbreak of group A streptococcal bacteremia in Sweden: an epidemiologic and clinical study. J Infect Dis 164:595-598

26. Tyrrell GJ, Lovgren M, Kress B, Grimsrud K (2005) Invasive group A streptococcal disease in Alberta, Canada (2000 to 2002). J Clin Microbiol 43:1678-1683 


\section{FIGURE LEGENDS}

1

2324

3

4325

5

6

8 9327 females

Fig. 1a Cases of invasive group A streptococcal disease during 1998-2007 in Finland. Thin line, number of cases; bold line, number of cases (12-week moving average)

Fig. 1b Monthly cases of iGAS during 1998-2007 in Finland (all years combined) in males and 
TABLES

1

2330

3

4

5

6

7

8

9

10

11

12

13

14

15

16

17

18

19

20

21

22

23

24

25

26

27

28

29

30

31

32

33

34333

35

36

37

38

39

40

41

42

43

44

45

46

47

48

49

50

51

52

53

54

55

56

57

58

59

60

61

62

63

64

65

Table 1 Annual incidence of invasive group A streptococcal disease and the number of cases and isolates studied, Finland, 1998-2007

\begin{tabular}{lcc}
\cline { 2 - 3 } Year & $\begin{array}{c}\text { Rate } \text { of invasive } \\
\text { disease }\end{array}$ & $\begin{array}{c}\text { Number of } \\
\text { cases (isolates) }\end{array}$ \\
\hline 1998 & 2.1 & 105 \\
1999 & 2.2 & 116 \\
2000 & 2.2 & 116 \\
2001 & 1.9 & 100 \\
2002 & 2.9 & 153 \\
2003 & 2.3 & 119 \\
2004 & 2.5 & $130(128)$ \\
2005 & 2.1 & $110(110)$ \\
2006 & 3.1 & $162(161)$ \\
2007 & 3.9 & $207(203)$ \\
All & 2.5 & $1318(602)$ \\
\hline
\end{tabular}

$332{ }^{a}$ Average annual incidence (cases per 100,000 population) 
334 Table 2 Age- and sex-specific incidence of invasive group A streptococcal disease, Finland, 1998-

\begin{tabular}{|c|c|c|c|c|c|c|c|c|}
\hline \multirow{3}{*}{$\begin{array}{l}\text { Age group } \\
\text { (years) }\end{array}$} & \multicolumn{6}{|c|}{ Rate $^{\mathrm{a}}$ of invasive disease (no. of cases) } & \multirow[b]{2}{*}{$\begin{array}{l}\text { Rate ratio } \\
\qquad(\mathrm{RR})^{\mathrm{b}}\end{array}$} & \multirow[b]{2}{*}{$\begin{array}{c}95 \% \mathrm{CI}^{\mathrm{c}} \text { of } \\
\mathrm{RR}\end{array}$} \\
\hline & \multicolumn{2}{|c|}{ Male } & \multicolumn{2}{|c|}{ Female } & \multicolumn{2}{|c|}{ Total } & & \\
\hline & 2.7 & (8) & 2.2 & $(6)$ & 2.5 & (14) & 1.3 & $0.4-3.9$ \\
\hline $1-14$ & 1.2 & $(51)$ & 0.9 & $(38)$ & 1.0 & $(89)$ & 1.3 & $0.8-2.0$ \\
\hline $15-24$ & 0.8 & $(26)$ & 1.2 & $(39)$ & 1.0 & $(65)$ & 0.6 & $0.4-1.0$ \\
\hline $25-34$ & 1.2 & (40) & 2.7 & (86) & 1.9 & (126) & 0.4 & $0.3-0.6^{*}$ \\
\hline $35-44$ & 3.0 & $(112)$ & 2.7 & $(97)$ & 2.8 & (209) & 1.1 & $0.9-1.5$ \\
\hline $45-54$ & 4.2 & $(168)$ & 1.8 & (69) & 3.0 & (237) & 2.4 & $1.8-3.2 *$ \\
\hline $55-64$ & 4.8 & (151) & 2.4 & (79) & 3.6 & (230) & 2.0 & $1.5-2.6^{*}$ \\
\hline$\geq 65$ & 5.1 & (163) & 3.8 & (185) & 4.3 & (348) & 1.4 & $1.1-1.7 *$ \\
\hline All & 2.8 & (719) & 2.2 & (599) & 2.5 & (1318) & 1.3 & $1.1-1.4^{*}$ \\
\hline
\end{tabular}

${ }^{\mathrm{a}}$ Average incidence (cases per 100,000 population)

$337{ }^{\mathrm{b}}$ male-to-female incidence rate ratio

31

32338

33

34339

35

36340

37

38

39

40

41

42

43

44

45

46

47

48

49

50

51

52

53

54

55

56

57

58

59

60

61

62

63 
341 Table 3 The number and proportion (\% of total number) of most common emm types encountered in 1342 the order of overall prevalence, and case fatality (\%) by emm type, 2004-2007, Finland.

\begin{tabular}{|c|c|c|c|c|c|c|c|c|c|c|c|c|}
\hline \multirow{3}{*}{$\begin{array}{l}\begin{array}{l}\text { emm } \\
\text { type }\end{array} \\
28\end{array}$} & \multicolumn{10}{|c|}{ Number of isolates $(\%)$} & \multirow{2}{*}{\multicolumn{2}{|c|}{$\begin{array}{c}\text { Fatal cases } \\
\qquad \%) \\
2004-2007 \\
(N=48)\end{array}$}} \\
\hline & \multicolumn{2}{|c|}{$\begin{array}{c}2004 \\
(\mathrm{~N}=128)\end{array}$} & \multicolumn{2}{|c|}{$\begin{array}{c}2005 \\
(\mathrm{~N}=110)\end{array}$} & \multicolumn{2}{|c|}{$\begin{array}{c}2006 \\
(\mathrm{~N}=161)\end{array}$} & \multicolumn{2}{|c|}{$\begin{array}{c}2007 \\
(\mathrm{~N}=203)\end{array}$} & \multicolumn{2}{|c|}{$\begin{array}{c}2004-2007 \\
(\mathrm{~N}=602)\end{array}$} & & \\
\hline & 46 & $(35.9)$ & 22 & $(20.0)$ & 32 & $(19.9)$ & 25 & $(12.3)$ & 125 & $(20.8)$ & 3 & (2) \\
\hline 1 & 6 & $(4.7)$ & 9 & $(8.2)$ & 26 & $(16.1)$ & 57 & $(28.1)$ & 98 & $(16.3)$ & 14 & (14) \\
\hline 84 & 0 & $(0.0)$ & 1 & $(0.9)$ & 24 & $(14.9)$ & 32 & $(15.8)$ & 57 & $(9.5)$ & 4 & (7) \\
\hline 75 & 10 & (7.8) & 17 & $(15.5)$ & 12 & $(7.5)$ & 4 & $(2.0)$ & 43 & (7.1) & 6 & (14) \\
\hline 89 & 10 & $(7.8)$ & 4 & $(3.6)$ & 11 & $(6.8)$ & 12 & $(5.9)$ & 37 & $(6.1)$ & 2 & $(5)$ \\
\hline 79 & 10 & $(7.8)$ & 12 & $(10.9)$ & 4 & $(2.5)$ & 2 & $(1.0)$ & 28 & $(4.7)$ & 4 & (14) \\
\hline 12 & 2 & (1.6) & 6 & $(5.5)$ & 6 & (3.7) & 12 & (5.9) & 26 & $(4.3)$ & 2 & (8) \\
\hline 81 & 7 & $(5.5)$ & 3 & (2.7) & 5 & (3.1) & 8 & (3.9) & 23 & (3.8) & 0 & (0) \\
\hline $27 \mathrm{G}$ & 3 & (2.3) & 1 & $(0.9)$ & 4 & $(2.5)$ & 11 & (5.4) & 19 & (3.2) & 2 & (11) \\
\hline other $^{\mathrm{a}}$ & 34 & $(26.6)$ & 35 & $(31.8)$ & 37 & $(23.0)$ & 40 & (19.7) & 146 & $(24.2)$ & $11^{\mathrm{b}}$ & (7) \\
\hline
\end{tabular}

$343{ }^{\text {a }}$ Including isolates from a total of 37 other emm types and nontypable isolates.

344 b Other types causing poor outcome: emm 4 and emm 110 ( 2 cases each); emm22, emm59, emm60, emm77, 345 emm 85 , emm87 and emm 112 (1 case each). 


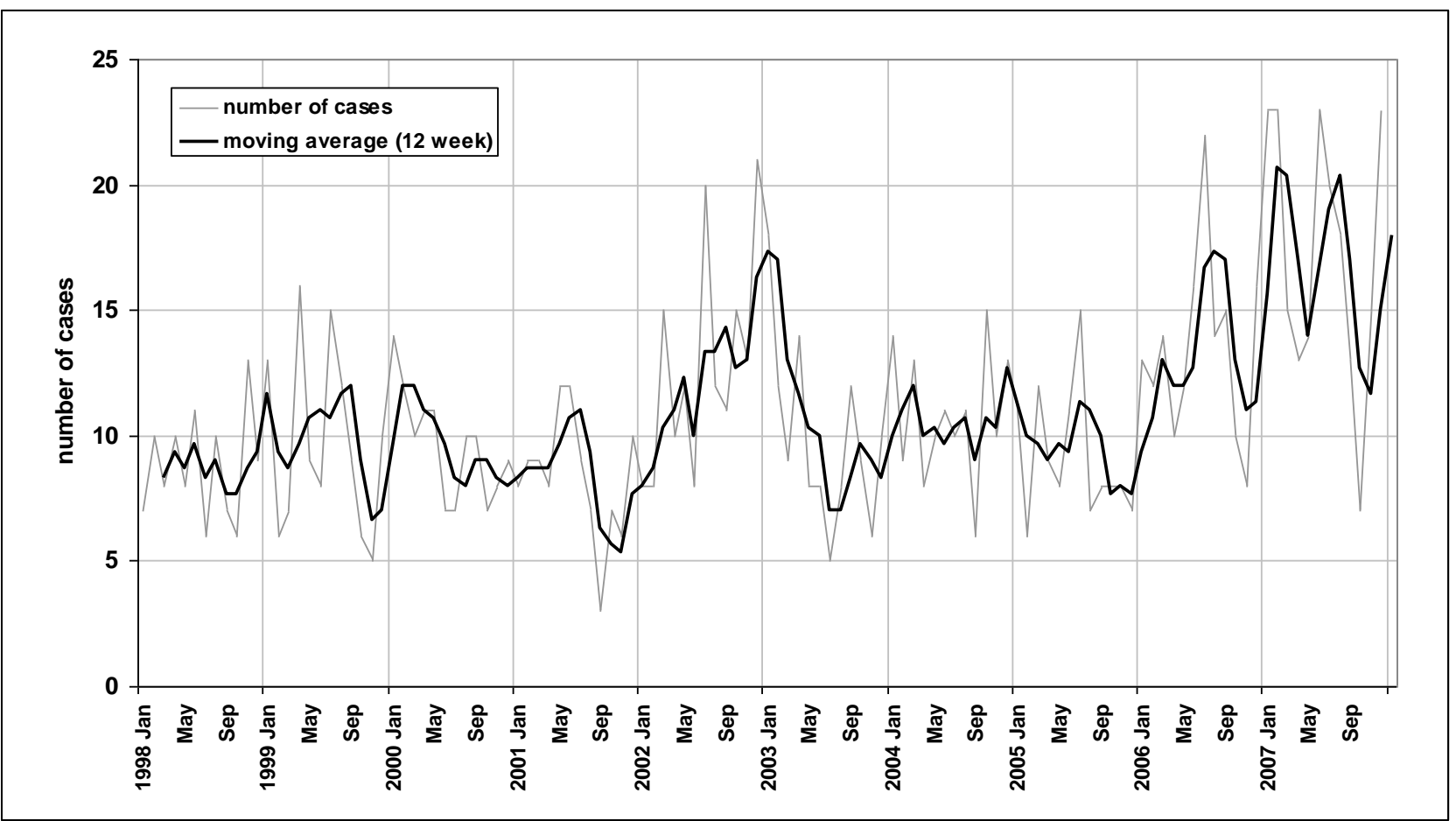

b

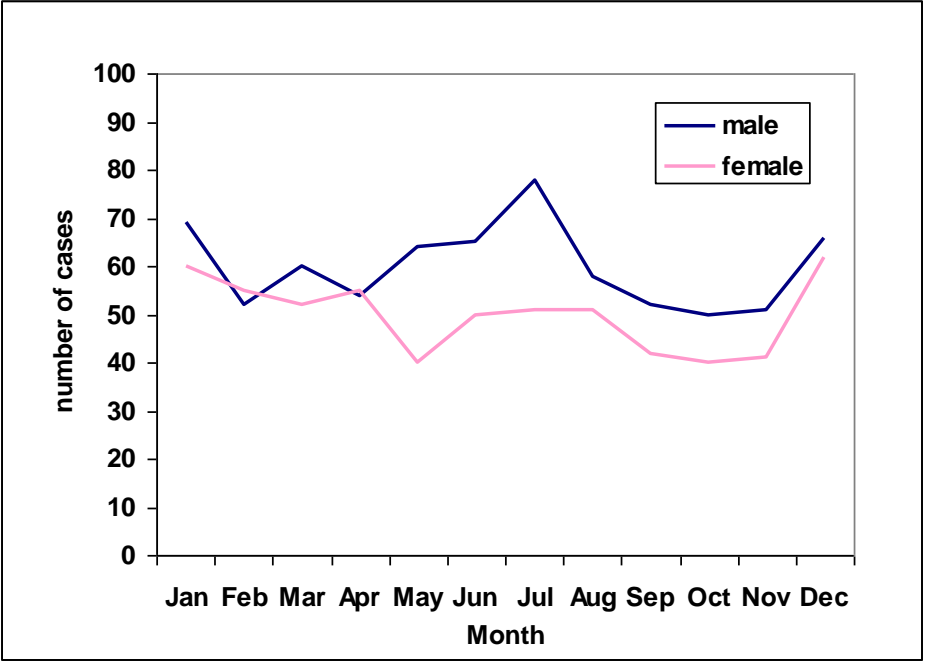

